\title{
POLÍTICAS PARA LA GESTIÒN DE LA INFORMACIÒN EN LA PLANIFICACIÒN TERRITORIAL
}

\author{
POLICIES FOR THE MANAGEMENT OF INFORMATION IN TERRITORIAL \\ PLANNING
}

\author{
LORENA VIVANCO CRUZ ${ }^{1}$, RAÚL MEJÍA ${ }^{2}$, VILLIE MOROCHO ${ }^{3}$
}

${ }^{1}$ UNIVERSIDAD DE CUENCA - FACULTAD DE ARQUITECTURA Y URBANISMO. ${ }^{2}$ PONTIFICIA UNIVERSIDAD CATÓLICA DEL ECUADOR - FACULTAD DE CIENCIAS EXACTAS Y NATURALES

${ }^{3}$ UNIVERSIDAD DE CUENCA - DEPARTAMENTO DE CIENCIAS DE LA COMPUTAICON, FACULTAD DE INGENIERÍA.

Universidad de Cuenca. Avenida 12 de Abril y Agustín Cueva, Cuenca, Ecuador

lorena.vivanco@ucuenca.edu.ec; raul.mejia@gmail.com; villie.morocho@ucuenca.edu.ec

Recibido: 24 de septiembre de 2018 / Aceptado: 4 de diciembre de 2018

\section{RESUMEN}

Este artículo, pone a debate la política de gestión de información en Ecuador, a partir del entendimiento de algunos conceptos desde tres puntos de vista - gestión del conocimiento, gestión de la información y gestión de contenidos-, para luego profundizar el marco normativo existente en materia de acceso y uso a la información pública en Ecuador, así como también analizar los sistemas de información geoespacial a nivel nacional y local, como una forma de abordar las políticas públicas. Más aún cuando, la disponibilidad de información territorial es necesaria para la gestión de los territorios, en diferentes escalas y entre diferentes niveles de Gobierno. Confirmando que la sola disponibilidad de datos no es suficiente, sobre todo cuando existen inconsistencias entre diferentes fuentes de información. Resulta común que para un mismo territorio existan datos que provienen de diferentes fuentes institucionales y que no son consistentes entre sí, en cuanto a la escala, detalle o fecha de adquisición. Esto provoca un problema a la hora de seleccionar una fuente de información, más aún cuando esa fuente institucional resulta ser una "copia parcial" o una "actualización" o "mejoramiento" de otra, volviendo incierta la "originalidad del dato". La implementación de una política nacional de "registros originales" puede ser la salida al desorden en las fuentes de información, como una política integral de gestión de la información pública, que mejorar la disponibilidad y consistencia de los datos que describen el territorio.

Palabras clave: gestión de la información; datos geoespaciales; territorio, planificación territorial, registros originales, sistemas de información territorial

\begin{abstract}
This article discusses the information management policy in Ecuador, based on the understanding of some concepts from three points of view - knowledge management, information management and content management-. After of this a review of the existing regulatory framework around of access and use of Ecuadorian public information is done. On the other hand, some local and national geospatial information systems are discussed, as a way to address public policies. Considering that the availability of territorial information is necessary for the territorial management, especially at different scales and different Government levels. It is confirming that isolated availability of data is not enough, especially when there are possible inconsistencies such as, for the same territory there are data that come from different institutional
\end{abstract}


sources and are not consistent with each other, in terms of scale, detail or acquisition date. This causes a problem in deciding "the best" source of information, finding even "partials copies" or an "updating" or "improvement" of another, which results in uncertain "originality of the data". The implementation of a national policy of "original records" may be the way out of the disorder in the sources of information, such as a comprehensive public information management policy, which will improve the availability and consistency of the data describing the territory.

Key words: land information systems, geospatial data, land use planning, key registers.

\section{INTRODUCCIÓN}

La gestión de la información para la planificación del territorio, según Alonso, Julio (2007), precisa diferenciar de partida tres conceptos, referidos a: "Datos. Se trataría de una medición objetiva; Información. Conjunto de datos relacionados e interpretados; Conocimiento. Conjunto de información desarrollada, que permite prever y planificar".

La comprensión de una política integral de gestión de la información pública, "que permitan transformar datos en información geoespacial relevante, para observar, experimentar, analizar y comprender el comportamiento del sistema territorial y urbano" (LaVCiTe. 2017), requiere considerar tres aspectos:

El primero, la gestión de la información, que define procesos y se refiere a la creación, captura, clasificación, recuperación y transferencia de la información que genera, recibe o adquiere una organización; lo que coincide con lo que sostiene Alonso, Julio (2007),que es la "explotación de la información para la consecución de los objetivos de la entidad. Su creación, adquisición, procesamiento y difusión".

El segundo, la gestión del conocimiento, que "vendría a ser el nivel superior, y estaría relacionado con las políticas de información, y además implicaría su asimilación por parte de los individuos que operan en la institución", (Alonso Julio, 2007); y, que además demanda una "arquitectura de información", sustentada en plataformas tecnológicas y sistemas de redes que posibiliten los flujos de intercambio de información y comunicación entre los individuos y los equipos para la realización de diversas actividades asociadas a los procesos de creación de conocimiento.

El tercero, la gestión de contenidos, que involucra una "serie de acciones y destrezas profesionales que permite la creación y administración de contenidos", (Alonso Julio, 2007), es decir la gestión de documentos y datos tanto internos como externos.

Para lograr una efectiva y coherente, gestión de información y el conocimiento, es necesario no solo emplear herramientas que permitan agilizar y optimizar los procesos, sino también conocer el marco jurídico de la gestión de información para la planificación del ordenamiento del territorio. Además, es primordial que éste cuente con políticas 
claras de producción, actualización, acceso y uso de los datos. Así como, políticas para la creación y gestión de metadatos, estándares para la información, junto con lineamientos sobre administración de tierras que describan su valor, propiedad y uso.

Todo proceso de gestión de información territorial como lo describe el Servicio Geológico Colombiano (2017), implica la, "planeación, adquisición, recibo, generación, administración, depuración, archivo, conservación, uso y difusión", entre otras características implícitas en un sistema de información, en el que interactúan individuos, información y los correspondientes procesos para generar un valor añadido a la misma. Un aspecto que surge con claridad en Ecuador es la necesidad de contar con políticas públicas que consideren la información de manera integral. Una forma de abordar las políticas públicas es la consideración de los marcos normativos existentes, como bien lo señala Natalia Torres (2014), "las leyes de acceso a la información imponen nuevas obligaciones a las administraciones que destacan la necesidad de una buena gestión de la información".

En este artículo hemos optado por relevar, exclusivamente los desarrollos regulatorios en materia de acceso y uso a la información pública en Ecuador, para luego analizar los sistemas de información geoespacial a nivel nacional y local, para proponer reflexiones y acciones hacia una política integral de gestión de la información pública.

\section{ORDENAMIENTO JURÌDICO PARA LA GESTIÒN DE LA INFORMACIÒN TERRITORIAL}

Respecto del desarrollo de la información territorial, Naciones Unidas (2015) señala que ésta "se constituye a partir del conjunto de datos fundamentales que comprende la información oficial del territorio para que cualquier usuario y aplicación pueda referenciar sus datos".

Algunas características de esta información son: diversidad (variedad de contenidos temáticos), calidad (actualización, atributos, escala), accesibilidad (inmediatez, de libre descarga), y usabilidad (mejor utilizable para darle un valor agregado, análisis territorial). Por lo tanto, se debe saber: ¿cómo se produce?, ¿Cómo se accede a ella? y ¿cuánto de pertinente es para determinados usos?.

Para establecer los requerimientos de la información territorial, es necesario conocer las "necesidades" y "los usuarios", como es el caso de los 1061 niveles de Gobiernos Autónomos Descentralizados -GAD-, los cuales, de acuerdo a la legislación nacional tienen la competencia de planificar el desarrollo y ordenamiento territorial. Otros usuarios lo constituyen también las Instituciones que tiene incidencia en la planificación del territorio incluyendo a los actores privados como son los consultores. 
La generación de la información territorial procede tanto del Estado como de la sociedad y requiere de tecnologías, personas, acuerdos organizativos, normas, entre otros, aspectos que lejos están de ser puramente informáticos. Tales requerimientos, plasman el valor social de la colaboración en la integración de la información territorial. Hernández, A. (2018), sostiene que el "valor social es entonces el resultado del acceso, pero sobre todo del tratamiento de la información con un objetivo concreto". Entonces, la información territorial proporcionada por las instituciones públicas tiene un valor social, y su utilidad radica en la utilidad que los ciudadanos le dan y no meramente en su carácter público. Por lo que, transparentar las acciones de gobierno, y en general cualquier ejercicio del poder público, facilita la rendición de cuentas y por lo tanto combate a la corrupción, en la medida que la ciudadanía tiene la posibilidad y las condiciones de analizar la información que de otro modo sería asequible únicamente a las élites técnicas y/o burocráticas. De los beneficios sociales, que se desprendan de esta evaluación depende que el derecho a la información pública se consolide en Ecuador, como un precepto constitucional.

Hernández, A. (2018) propone que "Las politicas de transparencia deben evaluarse en primer lugar por la oferta de información disponible y su calidad, y luego por la posibilidad de ésta de generar valor social. El carácter público de los datos no es suficiente al momento de procurar el derecho a la información, debe también promoverse que éstos puedan ser utilizados por cualquier ciudadano interesado". En el caso de Ecuador, es necesario entender que esa política de transparencia, se tipifica como un derecho constitucional, dado la posibilidad de que "Todas las personas, en forma individual o colectiva, tienen derecho a: El acceso universal a las tecnologías de información y comunicación", Constitución (2008). Además de quedar garantizado el acceso libre "a la información generada en entidades públicas, o en las privadas que manejen fondos del Estado o realicen funciones públicas", Constitución (2008).

Vale señalar que una de las políticas de transparencia y acceso a los datos, básica para la gestión de la información, lo constituyen la información que generan los sistemas de planificación y de finanzas públicas, que de acuerdo al Código Orgánico de Planificación y Finanzas Públicas -COPFP- (2010), "es de libre acceso, tanto para las personas naturales como para las jurídicas públicas y privadas".

La información para la planificación, tiene carácter oficialy es pública, y debe generarse y administrarse en función de las necesidades establecidas en los instrumentos de planificación, como son el Plan Nacional de Desarrollo, la Estrategia Territorial Nacional, Planes sectoriales, Planes estratégicos Nacionales, Planes de desarrollo y ordenamiento territorial de las regiones, provincias, cantones y parroquias rurales, entre otros definidos en la Ley, tanto para el ámbito supranacional, nacional, regional, provincial, can tonal, parroquial rural, regímenes especiales y otros instrumentos de planificación sectoriales, especiales y complementarios. No obstante, habrá de considerarse que la Secretaria Nacional de Planificación y Desarrollo, como ente rector 
de la planificación nacional, "define el carácter de oficial de los datos relevantes para la planificación nacional, y los lineamientos para la administración, levantamiento y procesamiento de la información, que serán aplicables para las entidades que conforman el sistema", a la vez que establece los mecanismos, metodologías y procedimientos aplicables a la generación y administración de la información para la planificación, así como sus estándares de calidad y pertinencia.

Es necesario señalar que tanto la "Norma Técnica para la creación, consolidación y fortalecimiento de los sistemas de información local", como las "Normas técnicas nacionales para el catastro de bienes inmuebles urbanos - rurales y avalúos de bienes; operación y cálculo de tarifas por los servicios técnicos de la dirección nacional de avalúos y catastros", no se han implementado en su integralidad. Particularmente las normas referidas al catastro de bienes inmuebles urbanos - rurales y avalúos de bienes, tiene a la fecha dos propuestas debido a la inaplicabilidad de la norma catastral.

En Ecuador, "el tratamiento de la información territorial", se sustenta en un marco regulatorio que considera al menos de manera directa las siguientes leyes: Ley de Transparencia y Acceso a la Información Pública -LTAIP-(2004); Constitución de la República de Ecuador -CRE-(2008); Código Orgánico de Planificación y Finanzas Públicas -COPFP- (2010); Ley Orgánica de Ordenamiento Territorial, Uso y Gestión de Suelo -LOOTUGS- (2016) y otras normas emitidas para el cumplimiento operativo de dichas leyes, tal como se indica en la siguientes figuras.

\begin{tabular}{|c|c|c|c|}
\hline \multirow{2}{*}{$\begin{array}{l}\text { Ley de Transparencia } \\
\text { y Acceso a la } \\
\text { Información Pública, } \\
\text {-I.TAIP- } \\
\qquad \begin{array}{l}2004\end{array}\end{array}$} & $\begin{array}{l}\text { Las personas, tienen } \\
\text { derecho a intercambiar, } \\
\text { producir y difundir } \\
\text { información }\end{array}$ & $\begin{array}{l}\text { Código Orgánico de } \\
\text { Planificación y Finamzas } \\
\text { Públicas, -COPFP- }\end{array}$ & $\begin{array}{l}\text { Obliga al Estado Central, gobiemos } \\
\text { locales y demás instituciones } \\
\text { relacionadas el ordenamiento del } \\
\text { territorio y a la ciudadania en } \\
\text { general, a remitir información. }\end{array}$ \\
\hline & & 2010 & \\
\hline & 2008 & & 2016 \\
\hline $\begin{array}{l}\text { Principio de } \\
\text { publicidad }\end{array}$ & $\begin{array}{l}\text { Constitución de la } \\
\text { República del Ecuador } \\
\text {-CRE- }\end{array}$ & $\begin{array}{l}\text { La información para la } \\
\text { planificación, tiene el } \\
\text { carácter oficial y público. }\end{array}$ & $\begin{array}{l}\text { Ley Orgánica de Ordenamiento } \\
\text { Territorial, Uso y Gestión de Suelo, } \\
\text { - LOOTUGS- }\end{array}$ \\
\hline
\end{tabular}

Figura 1. Marco regulatorio vigente para la información territorial en Ecuador, como principio constitucional

Fuente: LTAIP, 2004; CRE, 2008; COPFP, 2010; LOOTUGS, 2016. 


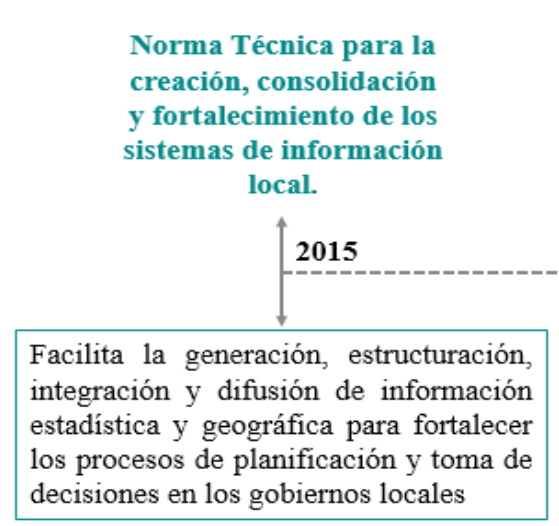

Norma Técnica para la creación, consolidación y fortalecimiento de los local.
Establece requisitos mínimos y criterios técnicos aplicables a la estructuración del Sistema Nacional de Catastro Integral Geo Referenciado de Hábitat y Vivienda.

\section{7}

Normas técnicas nacionales para el catastro de bienes inmuebles urbanos - rurales y avalúos de bienes; operación y cálculo de tarifas por los servicios técnicos de la dirección nacional de avalúos y catastros.

Figura 2. Normas complementarias para la información territorial en Ecuador. Fuente: Secretaria Nacional de Planificación y Desarrollo, 2015; Ministerio de Desarrollo Urbano y Vivienda, 2017.

Sin embargo, a pesar de tener un marco regulatorio vigente para la información territorial en Ecuador, existen algunos problemas que enfrenta, como: dispersión y fragmentación de los datos; información geográfica y estadística, limitada; escasa coordinación de la información territorial disponible; carencia de un sistema que integre datos e información estadística y geográfica relevante; duplicidad en las fuentes de datos, provocado por el desconocimiento de quien tiene la competencia del dato (registros originales); débiles mecanismos de homologación y estandarización de la información territorial; automatización incompleta; restringido acceso a los datos y pocas fuentes "originales" que liberan la información territorial con la que disponen ; y, escaso soporte técnico a las pocas fuentes que brindan servicios de información territorial. En un intento por estandarizar a cierto nivel la información espacial, se creó el Catalogo Nacional de Objetos Geográficos (CONAGE, 2015), sin que al momento haya conseguido su fin, pues son muy pocos los geoportales que evidencian el uso de dicho catálogo.

Sobre las Normas técnicas nacionales para el catastro de bienes inmuebles urbanos rurales y avalúos de bienes, es necesario señalar que este esfuerzo, por homologar la información para la planificación, no ha logrado concretarse en el país, debido a que tradicionalmente la normativa catastral trabajaba el catastro como insumo para la valoración de los bienes inmobiliarios y por ende del impuesto predial. En este sentido, la antigua Ley Orgánica de Régimen Municipal -LORM-, hace hincapié en el levantamiento de características de los predios que inciden en su valoración, y donde la geometría de los predios y sus construcciones se describen únicamente como áreas. Haciéndose nula referencia a la geometría y ubicación absoluta de los predios en un mapa. Esta ha sido la gestión tradicional de datos catastrales en el Ecuador. 
Más recientemente y desde la aparición de los computadores personales, a partir de la década de los 90, ciertos municipios, principalmente los de mayor tamaño como los de Quito, Guayaquil y Cuenca, incursionaron en describir a los predios no solamente con características de valoración y áreas de terreno y construcción, sino también con una representación geométrica, más específicamente de polígonos coincidentes con los linderos del terreno y algunos casos con los linderos del suelo ocupado con construcciones. Inicialmente mediante la utilización de aplicativos como los CAD (para diseño asistido por computadora) y actualmente mediante Sistemas de Información Geográfica -SIG-.

Sin embargo, la mayoría de municipalidades, continuaron cumpliendo mínimamente con lo requerido por la LORM y luego por el Código Orgánico de Organización Territorial, Autonomías y Descentralización -COOTAD-, es decir, el levantamiento de características prediales para valoración, y en pocos casos usando la geometría de los predios como instrumento accesorio sin valor legal. En este contexto, aparece una normativa con un fuerte enfoque instrumental, de carácter geográfico, y con algunas limitaciones conceptuales lo que dificulta su aplicación en los 221 municipios del país. Vale decir, que la normativa incluso llega a tratar temas demográfico-censales, que son totalmente ajenos al accionar de las municipalidades, aspecto que aleja aún más a los funcionarios municipales del cumplimiento de sus tareas de mantenimiento catastral.

Respecto de los Sistemas de Información Local, impulsados desde la Secretaría Nacional de Planificación y Desarrollo -SENPLADES-, estos han sido concebidos por las municipalidades como instrumentos de reporte, de su gestión sobre el territorio, de allí que un enfoque adecuado, sería el que el Gobierno Central impulse la construcción de estos sistemas como herramienta de gestión del territorio municipal, con una doble función, en primera instancia como instrumento de reporte, y luego como segundo paso, estaría el que esa herramienta plenamente instaurada en la gestión municipal pueda ser usada por el gobierno central. Pues, tal como se emite esta normativa, se interpreta que es una herramienta que debe ser construida por las municipalidades para beneficio del gobierno central únicamente.

\section{ANÁLISIS DE LOS SISTEMAS DE INFORMACIÓN NACIONALES Y LOCALES EN ECUADOR}

Dagnino, J. (2010), señala que "la información puede mejorar el bienestar individual y social [...]", desde una visión dinámica, induciendo progresos futuros referidos a:

“a) Desplegando la función de utilidad: ampliando las dimensiones y las magnitudes del bienestar.

b) Expandiendo la frontera de posibilidades de producción: mejorando la calidad de los factores de producción, incluso de la tecnología, de las instituciones y de aspectos sociales, $y$ su uso.

c) Ampliando las interfaces entre la producción y el consumo: creando más posibilidades de comercio y de financiamiento", a través de la educación, la difusión tecnológica y el 
funcionamiento institucional, lo cual disminuye la incertidumbre y el riesgo presente en la toma de decisiones que la ciudadanía, políticos, académicos, planificadores y otros, hacen respecto de los procesos de planificación del territorio.

La nueva tendencia de ciudades inteligentes, coloca al ciudadano en el centro del desarrollo, incorporando las TICs como un elemento primordial para la gestión urbana. Dichos elementos deberían ser utilizados como herramientas para conseguir el diseño de un gobierno efectivo que incluya la planeación colaborativa y la participación ciudadana. Ello porque, una ciudad inteligente se vuelve más innovadora, competitiva, atractiva y resiliente, mejorando la calidad de vida de sus ciudadanos.

La producción y consumo de la información territorial debe obligatoriamente, además de ser pública, provenir de los "registros originales". Esto implica que un dato está certificado, se recolecta una sola vez, y una entidad del gobierno -ente rector o GAD, es el responsable de producir, actualizar y mantener el dato.

En base a la legislación ecuatoriana, la competencia del dato está en varias entidades, de acuerdo al ámbito de sus competencias establecidas en la Constitución y otras leyes. Por ejemplo, los gobiernos autónomos descentralizados regionales (aún no conformadas) delimitan los ecosistemas de escala regional y las cuencas hídricas, en coordinación con la Secretaria Nacional del Agua (como autoridad única del agua). Los gobiernos autónomos descentralizados provinciales, son responsables de definir la vialidad provincial - es decir la infraestructura vial rural. En tanto que, a las municipalidades les corresponde definir la vialidad urbana. Mientras al Ministerio de Transporte y Obras Públicas -MTOP-, la red vial estatal. Finalmente, los gobiernos autónomos descentralizados municipales y metropolitanos definen la clasificación del suelo en urbano y rural, entre otros aspectos.

En base a la competencia del dato y de acuerdo a la legislación nacional, existen algunos "sistemas" albergados en Instituciones Públicas, que proveen de datos estadísticos y geográficos a los procesos de planificación, entre ellos se puede citar:

Sistema Estadístico y Geográfico Nacional (INEC): "es la fuente de información para el análisis económico, social, geográfico y ambiental, que sustente la construcción y evaluación de la planificación de la política pública en los diferentes niveles de gobierno.

La información estadística y geográfica que cumpla con los procedimientos y normativa establecida, tendrá el carácter de oficial y deberá ser obligatoriamente entregada por las instituciones integrantes del Sistema Estadístico Nacional al organismo nacional de Estadística para su utilización, custodia y archivo". (Artículo 32 del Código Orgánico de Planificación y Finanzas Públicas, 2010).

Sistema Nacional de Información -SNI- (SENPLADES): "constituye el conjunto organizado de elementos que permiten la interacción de actores con el objeto de 
acceder, recoger, almacenar y transformar datos en información relevante para la planificación del desarrollo y las finanzas públicas. Sus características, funciones, fuentes, derechos y responsabilidades asociadas a la provisión y uso de la información serán regulados por este código, su reglamento y las demás normas aplicables.

La información que genere el Sistema Nacional de Información deberá coordinarse con la entidad responsable del registro de datos y la entidad rectora de las finanzas públicas, en lo que fuere pertinente". (Artículo 33 del Código Orgánico de Planificación y Finanzas Públicas, 2010).

Sistema de Información Local (Gobiernos Autónomos Descentralizados): “es el conjunto organizado y sistemático de elementos,-dependencias técnicas y administrativas; talento humano; medios técnicos; procedimientos en general; productos informativos- que permitan la interacción de los Gobiernos Autónomos Descentralizados con la ciudadanía en el territorio, en el marco de la rendición de cuentas y control social; para acceder, recoger, almacenar, transformar y difundir datos en información relevante para la planificación, el desarrollo y la gestión pública, local y nacional". (Secretaria Nacional de Planificación y Desarrollo. Reforma a la Norma Técnica para la creación, consolidación y fortalecimiento de los Sistemas de Información Local, 2018).

Sistema de Información Pública Agropecuaria (Ministerio deAgricultura y Ganadería): tiene por objeto "generar, administrar y proveer información oportuna a los productores y agentes económicos que participan en la producción y en los mercado s agropecuarios y de servicios relacionados con la tierra rural. En este sistema se integrará información relativa a:

a) Aspectos económicos relevantes de la actividad agro productiva, desarrollo rural y de la soberanía alimentaria;

b) Aptitud y uso de la tierra rural;

c) Niveles de productividad, fertilidad y vulnerabilidad del suelo;

d) Información relativa a la oferta y demanda de tierra rural y de agua para riego;

e) Tipo de propiedad y forma de adquisición;

f) Estadística agropecuaria;

g) Condiciones climatológicas;

h) Comercialización de la producción agropecuaria y precios;

i) Márgenes de intermediación comercial relativos a la agricultura familiar campesina;

j) Contratos agrícolas, modalidades de arrendamiento y agricultura por contrato;

k) Fuentes y condiciones de crédito, estructura de distribución del crédito agropecuario;

1) Infraestructura rural: movilidad, riego, acopio y comercialización;

m) Cartografía;

n) Tecnología y servicios técnicos;

o) Catastro rural; y,

p) Otros temas que requiera la integralidad del sistema. 
La Autoridad Agraria Nacional, en coordinación con la Autoridad del Sistema Nacional de Registro de Datos Públicos y los Gobiernos Autónomos Descentralizados, implementará y mantendrá actualizado este sistema de conformidad con la Ley", (Artículo 41 de la Ley Orgánica de Tierras Rurales y Territorio Ancestrales, 2016).

Sistema Nacional de Información Municipal (Asociación de Municipalidades Ecuatorianas): es una plataforma virtual de acceso restringido que contiene información relativa a: agua potable y alcantarillado gestión integral de residuos sólidos, cooperación internacional, tránsito y transporte.

Sistema Nacional de Administración de Tierras -SINAT-, (Gobiernos Autónomos Descentralizados Municipales): es una solución informática innovadora diseñada para la gestión catastral de los predios rurales. "El SINAT, conforme las políticas nacionales, está desarrollado en software libre, es administrado a nivel local y nacional, y está enfocado principalmente en gestionar los trámites catastrales necesarios para obtener la emisión predial con fines tributarios de conformidad con la legislación vigente". (Ministerio de Agricultura y Ganadería, 2018).

Sistema Nacional de Catastro Integral Georreferenciado (Ministerio de Desarrollo Urbano y Vivienda -MIDUVI-): "Es un sistema de información territorial generada por los Gobiernos Autónomos Descentralizados municipales y metropolitanos, y las instituciones que generan información relacionada con catastros y ordenamiento territorial, multifinalitario y consolidado a través de una base de datos nacional, que registrará en forma programática, ordenada y periódica, la información sobre los bienes inmuebles urbanos y rurales existentes en su circunscripción territorial.

El Catastro Nacional Integrado Georreferenciado deberá actualizarse de manera continua y permanente, y será administrado por el ente rector de hábitat y vivienda, el cual regulará la conformación y funciones del Sistema y establecerá normas, estándares, protocolos, plazos y procedimientos para el levantamiento de la información catastral y la valoración de los bienes inmuebles tomando en cuenta la clasificación, usos del suelo, entre otros. Asimismo, podrá requerir información adicional a otras entidades públicas y privadas. Sus atribuciones serán definidas en el Reglamento de esta Ley.

La información generada para el catastro deberá ser utilizada como insumo principal para los procesos de planificación y ordenamiento territorial de los Gobiernos Autónomos Descentralizados municipales y metropolitanos, y alimentará el Sistema Nacional de Información", (Artículo 100 de la Ley Orgánica de Ordenamiento Territorial, Uso y Gestión de Suelo, 2016).

Sistema Único de Información Ambiental -SUIA- (Ministerio del Ambiente), es una aplicación WEB que fue desarrollada para integrar toda la información ambiental en un solo lugar para generar indicadores geográficos, documentales, estadísticos 
y automatización de procesos institucionales, encaminados al control, registro, mantenimiento y preservación del medio ambiente.

Aunque estos sistemas son los más relacionados con la planificación del territorio, existen otros sistemas y entidades que generan información territorial, tal es el caso de algunos institutos de investigación, pero con acceso restringido o de uso interno para la entidad académica.

Actualmente todos los sistemas descritos funcionan, exceptuando el Sistema Nacional de Catastro Integral Georreferenciado y el Sistema de Información Pública Agropecuaria, que aunque están establecidos por ley, aún no han sido conformados en su integralidad.

La gestión de la información, en cada uno de los sistemas descritos, presenta algunas debilidades que deben ser consideradas por aquellas entidades que están encargadas de certificar los datos, con el objeto de mejorar las normas y estándares para implementar y fortalecer los sistemas de información geoespacial para los procesos de planificación de los diferentes niveles de gobiernos autónomos descentralizados del país. Dichas debilidades son:

El territorio es un tema integral, que por su carácter puede incluir un sinnúmero de aspectos, variables e indicadores, por lo tanto los sectores o entidades, que lo planifican, deben tener presente que el territorio es uno solo, por lo que no es necesario dividirlo en dos: el "geográfico" y el "estadístico".

El catastro no es una temática puramente informática, ni tampoco cartográfica, es necesario superar la interpretación instrumental por una más conceptual. Por ejemplo, ver al catastro como una descripción de la fragmentación de la tierra, lo cual lo hace invaluable en infinidad de aplicaciones. La ejecución del catastro es competencia municipal, no del gobierno central, aunque la regulación sí la sea.

No excluir de los sistemas de información territorial a aquellos datos que no son considerados "cartográficos", es así que resulta mucho más integrador el usar el concepto de información geoespacial, donde datos con un componente geoespacial son bien recibidos: direcciones, tabulaciones demográficas entre otros.

En un intento de "espacialización" de datos, muchas entidades asumen como suyos datos que originalmente provienen de otras instituciones. Es así que se genera una suerte de "colición" entre fuentes de información, solo una entidad será considerada el registro original.

El INEC, no ha "espacializado" sus estadísticas a pesar de que todas tienen una ubicación en el territorio (coropletas). El INEC dispone de mucha información "geoespacial", donde el componente espacio está bien determinado y delimitado por las diferentes polígonos de la división política administrativa. 
Cada institución asume de forma obligatoria la "competencia del dato" (registros originales), misma que estará muy ligada a las competencias institucionales.

\section{CONCLUSIONES}

Disponer de información oficial y registros originales, sirve para reconocer y expresar los fenómenos y estructuras que se dan sobre un territorio especifico, vale decir "explicar el desarrollo territorial, [...] de una manera inteligente", (Vries, 2009). Entonces, para mejorar la arquitectura interorganizacional de los sistemas de información territorial de los Gobiernos Autónomos Descentralizados y del Estado Central, es necesario trabajar con los registros o fuentes originales prescindiendo de copias en las diferentes instituciones, esto como una forma de inteligencia territorial. Un gobierno moderno y dinámico, nunca pregunta dos veces sobre un dato, menos aún lo almacena dos veces, con un entramado de registros originales, de los diferentes aspectos del territorio puede estar bien enterado, por lo tanto es eficiente.

La necesidad de información territorial, no es exclusiva de los tomadores de decisiones, sino también de otros actores del sistema, de forma individual u organizada, ya que sirve para orientar el diseño, ejecución y evaluación de las dinámicas y procesos de planificación. En este sentido la academia, puede ser la institución encargada de integrar la información autónoma, como lo ha hecho la Universidad de Cuenca, a través de la plataforma del Laboratorio Virtual de Ciudad y Territorio -LaVCiTe- en un intento por superar las limitaciones de los diferentes entes generadores de información e integrarlos manteniendo la responsabilidad sobre la originalidad del dato en la institución generadora. Sin duda, estas iniciativas académicas incorporan tecnologías de la información y comunicación para afrontar retos urbanos, complejos y persistentes.

La posibilidad de que la información sea accesible a la ciudadanía, y tenga el carácter oficial y público, como precepto constitucional, es el primer paso hacia la generación de una política integral de gestión de la información pública, pero también la implementación de una política nacional de "registros originales" puede ser la salida al desorden en las fuentes de información, aspecto que está muy relacionado con las competencias institucionales. Entonces una política adecuada para la gestión de información, lo constituye la originalidad del dato como una política a mejorar la disponibilidad y consistencia de los datos que describen el territorio.

\section{AGRADECIMIENTOS}

Este proyecto ha sido financiado por la Universidad de Cuenca - Facultad de Arquitectura y Urbanismo. 


\section{REFERENCIAS}

Asamblea Nacional (2015). Constitución de la República del Ecuador. Incluye las reformas aprobadas en el Referéndum y Consulta Popular de 7 de mayo de 2011 y las Enmiendas Constitucionales publicadas en el Registro Oficial No. 653 del 21 de diciembre de 2015.

Asamblea Nacional (2010). Código Orgánico de Organización Territorial, Autonomía y Descentralización. Registro Oficial No 303.

Asamblea Nacional (2010). Código Orgánico de Planificación y Finanzas Públicas. Registro Oficial No 306.

Asamblea Nacional (2016). Ley Orgánica de Ordenamiento Territorial, Uso y Gestión de Suelo. Registro Oficial No 790 .

Asamblea Nacional (2016). Ley Orgánica de Tierras y Territorios Ancestrales. Registro Oficial $\mathrm{N}^{\mathrm{o}} 711$.

Alfonso Arévalo, J. (2007). Gestión de información, gestión de contenidos y conocimiento. Recuperado a partir de http://eprints.rclis.org/11273/1/Jornadas_GRUPO_SIOU.pdf

Asociación de Municipalidades Ecuatorianas (2018). Sistema Nacional de Información Municipal. Recuperado a partir de http://www.snim.ame.gob.ec/

Catálogo Nacional de Objetos Geográficos. V2.0 - Ambar DataStore. (s. f.). Recuperado 21 de septiembre de 2018, de http://ambar.utpl.edu.ec/es/dataset/catalogo-nacional-de-objetosgeograficos-v2-0

Hernández, A. (2018). El valor social de la información. Recuperado a partir de https://www. jornada.com.mx/2018/07/25/opinion/018a1pol

Ministerio del Ambiente (2018). Sistema Único de Información Ambiental (SUIA). Recuperado a partir de http://www.ambiente.gob.ec/sistema-unico-de-informacion-ambiental-suia/

Ministerio de Agricultura y Ganadería (2018). Sistema Nacional para la Administración de Tierras (SINAT). Recuperado a partir de http://www.sigtierras.gob.ec/sistema-nacionalpara-la-administracion-de-tierras-sinat/

Secretaria Nacional de Planificación y Desarrollo (2018). Acuerdo Ministerial No. SNPD-0062018.

Servicio Geológico Colombiano (2017). Políticas de gestión de la información geo científica. Proceso gestión del conocimiento geo científico. Recuperado a partir de https://www2. sgc.gov.co/sgc/mapas/Documents/PDF\%20POL\%C3\%8DTICAS/politicas-gestioninformacion-dgi0.pdf

Torres, N. (2014). Hacia una política integral de gestión de la información pública. Recuperado a partir de https://www.palermo.edu/cele/pdf/Hacia_una_politica_integral-kk.pdf

Vries, A. (2009). Gestión de información territorial para la toma de decisiones en Cohesión e Inteligencia Territorial (pp. 139-152). 